\title{
THE FAINT END OF THE CLUSTER GALAXY LUMINOSITY FUNCTION AT HIGH REDSHIFT
}

\author{
Conor L. Mancone ${ }^{1}$, Troy Baker ${ }^{1}$, Anthony H. Gonzalez ${ }^{1}$, Matthew L. N. Ashby ${ }^{2}$, Spencer A. Stanford ${ }^{3,4}$, \\ Mark Brodwin ${ }^{5}$, Peter R. M. Eisenhardt ${ }^{6}$, Greg Snyder $^{2}$, Daniel Stern $^{6}$, Edward L. Wright ${ }^{7}$
}

\begin{abstract}
We measure the faint end slope of the galaxy luminosity function (LF) for cluster galaxies at $1<z<1.5$ using Spitzer IRAC data. We investigate whether this slope, $\alpha$, differs from that of the field LF at these redshifts, and with the cluster LF at low redshifts. The latter is of particular interest as low-luminosity galaxies are expected to undergo significant evolution. We use seven highredshift spectroscopically confirmed galaxy clusters drawn from the IRAC Shallow Cluster Survey to measure the cluster galaxy LF down to depths of $M^{*}+3(3.6 \mu \mathrm{m})$ and $M^{*}+2.5(4.5 \mu \mathrm{m})$. The summed LF at our median cluster redshift $(z=1.35)$ is well fit by a Schechter (1976) distribution with $\alpha_{3.6 \mu \mathrm{m}}=-0.97 \pm 0.14$ and $\alpha_{4.5 \mu \mathrm{m}}=-0.91 \pm 0.28$, consistent with a flat faint end slope and is in agreement with measurements of the field LF in similar bands at these redshifts. A comparison to $\alpha$ in low-redshift clusters finds no statistically significant evidence of evolution. Combined with past studies which show that $M^{*}$ is passively evolving out to $z \sim 1.3$, this means that the shape of the cluster LF is largely in place by $z \sim 1.3$. This suggests that the processes that govern the build up of the mass of low-mass cluster galaxies have no net effect on the faint end slope of the cluster LF at $z \lesssim 1.3$.

Subject headings: galaxies: clusters: general, galaxies: evolution, galaxies: formation, galaxies: luminosity function
\end{abstract}

\section{INTRODUCTION}

Many studies have shown that the low-mass clustergalaxy population evolves substantially at low redshift. For instance, Cowie et al. (1996) first recognized that star formation happens primarily in high-mass systems at high redshift and low-mass systems at low redshift, a fact which has been studied extensively since (see, for example, Panter et al. 2007; Mobasher et al. 2009; Chen et al. 2009; Villar et al. 2011). Moreover, it is well known that cluster galaxies undergo morphological transformation at low redshift, with many cluster members transforming to lenticular galaxies at low redshift (Dressler et al. 1997; Desai et al. 2007; Wilman et al. 2009). There is also substantial evidence that the low-luminosity redsequence galaxy population grows substantially in clusters since at least $z \sim 1$ (Stott et al. 2007; Lu et al. 2009; Rudnick et al. 2009; Lemaux et al. 2012).

Taken together, these facts demonstrate that the lowmass cluster galaxies are actively evolving and forming since $z \sim 1$. Therefore, by comparing low mass, $z=0$ galaxies with their high-redshift progenitors we can potentially constrain the processes important in galaxy formation and evolution. This can be done by studying individual galaxies (through their star formation rates, stellar masses, morphological types, and structural prop-

Electronic address: cmancone@astro.ufl.edu

1 Department of Astronomy, University of Florida, Gainesville, FL 32611

2 Harvard-Smithsonian Center for Astrophysics, 60 Garden Street, Cambridge, MA 02138

3 Physics Department, University of California, Davis, CA 95616

${ }^{4}$ Institute of Geophysics and Planetary Physics, Lawrence Livermore National Laboratory, Livermore, CA 94550

${ }^{5}$ Department of Physics and Astronomy, University of Missouri, 5110 Rockhill Road, Kansas City, MO 64110

6 Jet Propulsion Laboratory, California Institute of Technology, 4800 Oak Grove Drive, Pasadena, CA 91109

7 UCLA Astronomy, P.O. Box 951547, Los Angeles, CA 900951547 erties) or by studying galaxy populations (through their luminosity and mass functions).

In particular, the near-infrared luminosity function (NIR LF) can be used to study the stellar mass growth of a galaxy population, as the rest-frame NIR is a good proxy for stellar mass (Muzzin et al. 2008). In clusters, the NIR LF has been used extensively to study the assembly of the most massive cluster galaxies. Such studies have found that the massive end of the NIR LF evolves passively out to $z \sim 1.3$, suggesting that the bulk of the stellar mass of these galaxies is in place at high redshift (Andreon 2006; Strazzullo et al. 2006; De Propris et al. 2007; Muzzin et al. 2008; Mancone et al. 2010). In addition, in Mancone et al. (2010) we found statistically significant deviations from passive evolution at $z>1.3$ which we could only explain with ongoing stellar mass assembly at these redshifts.

Most attempts to probe the faint end of the cluster luminosity function (LF) at high redshift have been limited to studying the red sequence. Such studies have found a deficit of faint and red cluster members at high redshift when compared to their low-redshift counterparts (De Lucia et al. 2004; Stott et al. 2007; Lu et al. 2009; Rudnick et al. 2009; Lemaux et al. 2012). This could mean that low-mass cluster galaxies undergo substantial mass growth at low redshift, or simply that low-mass cluster galaxies are still blue at high redshift and have not finished transitioning onto the red sequence (Lemaux et al. 2012). Differentiating between these two cases requires measuring the LF of all faint cluster members. Previously, Strazzullo et al. (2010) was the only study to do this, finding a faint-end slope consistent with flat. However, they did not compare their results to low-redshift clusters to determine the implications for the stellar mass growth of low-mass cluster galaxies.

In this paper we measure the 3.6 and $4.5 \mu \mathrm{m} \mathrm{LF}$ of high redshift $(1<z<1.5)$ galaxy clusters. Our measure- 
TABLE 1

Cluster Member Summary

\begin{tabular}{ccccc}
\hline \hline Cluster & RA & Dec & z & \# Members \\
\hline ISCS J1432.4+3332 & $14: 32: 29.18$ & $33: 32: 36.0$ & 1.112 & 26 \\
ISCS J1434.5+3427 & $14: 34: 30.44$ & $34: 27: 12.3$ & 1.238 & 19 \\
ISCS J1429.3+3437 & $14: 29: 18.51$ & $34: 37: 25.8$ & 1.261 & 18 \\
ISCS J1432.6+3436 & $14: 32: 38.38$ & $34: 36: 49.0$ & 1.351 & 12 \\
ISCS J1433.8+3325 & $14: 33: 51.13$ & $33: 25: 51.1$ & 1.369 & 6 \\
ISCS J1434.7+3519 & $14: 34: 46.33$ & $35: 19: 33.5$ & 1.374 & 10 \\
ISCS J1438.1+3414 & $14: 38: 08.71$ & $34: 14: 19.2$ & 1.414 & 16 \\
\hline
\end{tabular}

ments trace the rest-frame NIR, where the LF is known to correlate well with stellar mass. Most importantly, our data are deep enough to constrain $\alpha$, the faint-end slope of the LF. This, combined with low-redshift results from the literature, allows us to measure the stellar mass buildup of the low-mass cluster galaxy population over a redshift range when these galaxies are known to be actively evolving.

This paper is structured as follows. Section 2 describes our data. Section 3 presents our method for measuring the galaxy cluster luminosity function and gives our results. In Section 4 we compare our results to low-redshift clusters and the field. Our conclusions are presented in Section 5. All magnitudes are on the Vega system, and we assume a WMAP 7 cosmology (Komatsu et al. 2011; $\left.\Omega_{m}=0.272, \Omega_{\Lambda}=0.728, h=0.704\right)$ throughout. All SPS model predictions are generated using EzGal (Mancone \& Gonzalez 2012).

\section{DATA}

\subsection{Cluster Sample}

The clusters from this study are part of the IRAC Shallow Cluster Survey (ISCS) (Stanford et al. 2005; Elston et al. 2006; Brodwin et al. 2006; Eisenhardt et al. 2008), a catalog of clusters identified as 3 -D overdensities using photometric redshifts in the $8.5 \mathrm{deg}^{2}$ Boötes field. Further work with the high-redshift $(1<z<1.5)$ clusters in the ISCS has included deep (1000s) IRAC imaging, spectroscopic followup, and HST imaging. Seven of the spectroscopically confirmed, high-redshift ISCS clusters have both deep IRAC imaging and ACS F775W imaging. It is this subsample of ISCS clusters that we use to study the LF of high-redshift galaxy clusters. We supplement the 1000 seconds of targeted IRAC observations for each cluster with imaging from the Spitzer Deep, Wide-Field Survey (SDWFS, Ashby et al. 2009) which has a median exposure time of 420 seconds throughout the Boötes field. This gives a total observing time of roughly $1400 \mathrm{~s}$ per cluster in all four IRAC bands.

We list in Table 1 our clusters along with their positions, redshifts and number of spectroscopic members. ISCS J1438.1+3414 has a published X-ray mass estimate of $\log \left(M_{200}^{L_{X}} / M_{\odot}\right)=14.35_{-0.14}^{+0.11}$ which comes from a 143 ks Chandra exposure (Andreon et al. 2011; Brodwin et al. 2011). All of these clusters (with the exception of ISCS J1433.8+3325) have a weak-lensing mass estimate from Jee et al. (2011), with masses in the range of $14.40 \leq \log \left(M_{200}^{W L} / M_{\odot}\right) \leq 14.73$.

\subsection{Comparison Fields}

Given the limited spectroscopic redshifts in these fields, statistical background subtraction is required to recover the underlying LF. A statistical background subtraction involves measuring the number counts of galaxies in a field region and subtracting it from the number counts of galaxies near the cluster. This technique has been used successfully by de Propris et al. (1998), Lin et al. (2004), Muzzin et al. (2008), and Mancone et al. (2010). This requires a survey with IRAC imaging of at least the same depth as our cluster images as well as ACS F775W imaging. For this purpose we select the GOODS North and South (Dickinson et al. 2003) fields. We downloaded the latest fully reduced 3.6 and $4.5 \mu \mathrm{m}$ Spitzer IRAC images taken of the GOODS fields. We also retrieved the latest HST ACS F775W catalogs from the GOODS survey. Throughout this paper we refer to the GOODS fields as our control fields.

\subsection{Data Reduction and Processing}

We produced IRAC mosaics of all seven clusters by combining data from our own programs (PID78, PID30950) with that from SDFWS, following procedures identical to those described in Ashby et al. (2009). This included the manner in which outliers were rejected and in the way the individual IRAC frames were prepared for mosaicing by first removing the residual images arising from earlier exposure to bright sources. We generated catalogs by running our fully reduced 3.6 and $4.5 \mu \mathrm{m}$ IRAC images (for the clusters and the control fields) through Source Extractor (Bertin \& Arnouts 1996) in single-image mode. We used 4/ diameter aperture mags that were aperture-corrected to total mags by comparing 4/I and 24// diameter aperture magnitudes for bright, unsaturated stars in our images. We used stars to measure the aperture corrections because galaxies at these redshifts are typically unresolved in IRAC imaging. This gave aperture corrections of $-0.32(-0.34)$ magnitudes in $3.6(4.5) \mu \mathrm{m}$ for our cluster images and $-0.31(-0.32)$ mags for our control images. For reference, the difference between the aperture corrections of the cluster and control fields is smaller than the uncertainty of the absolute flux calibration for IRAC images (Reach et al. 2005). To verify our calculated aperture corrections we compared our 4/l aperture corrected magnitudes to the $4 / \prime$ aperture corrected magnitudes from SDWFS, and found very small systematic offsets $(<0.03$ mags $)$.

The ACS imaging for our clusters was obtained as part of the HST Cluster Supernova Survey, and the reduction of the images is described in detail in Suzuki et al. (2012). We ran the reduced ACS F775W images through Source Extractor and used MAG_AUTO to calculate the F775W 


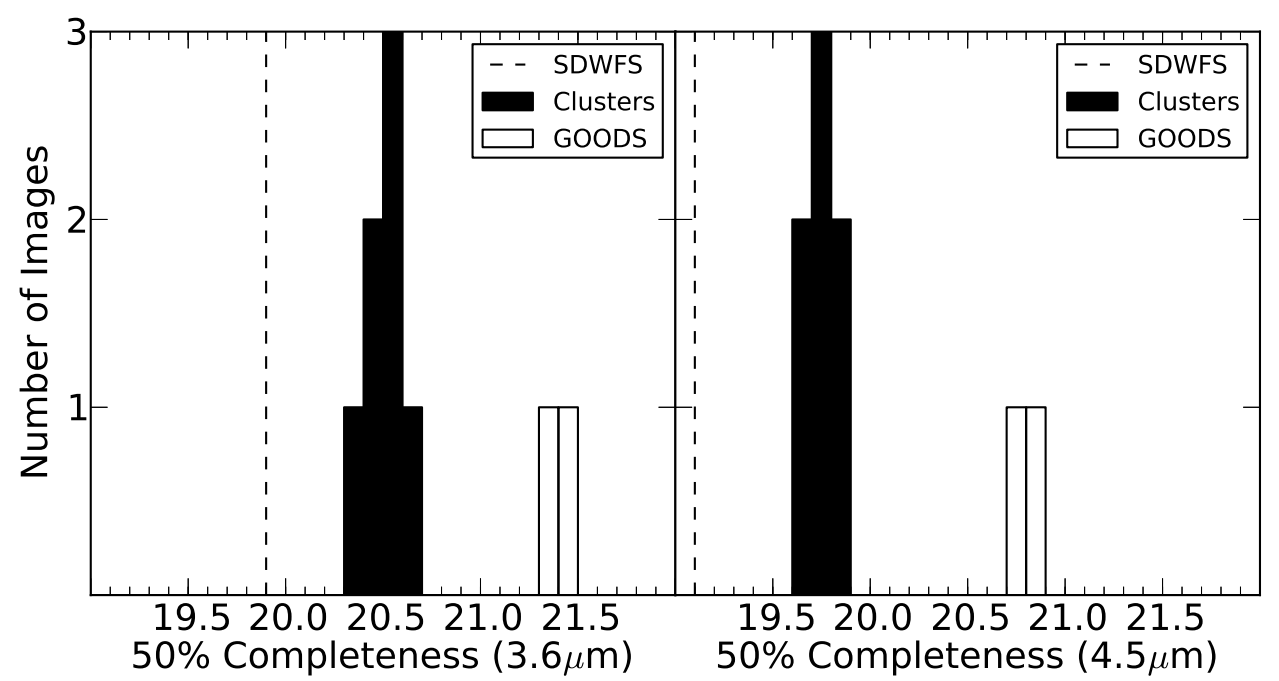

FIG. 1. - Measured $50 \%$ completeness limits for our images in 3.6 (left) and $4.5 \mu \mathrm{m}$ (right). In both panels the solid histograms show the completeness limits for our cluster fields, and open histograms show completeness for our control fields. The vertical dashed line denotes the $50 \%$ completeness limits from the SDWFS survey (Ashby et al. 2009).

magnitude of our galaxies. We used Our F775W photometry to perform an optical-NIR color cut to remove contaminants from our LF (Section 3.1). For our control we used the ACS F775W MAG_AUTO values from the GOODS catalogs, which were also generated with Source Extractor.

Next we calculated completeness as a function of magnitude at 3.6 and $4.5 \mu \mathrm{m}$ for each cluster image and each control image separately. We approximated our galaxies as point sources due to the coarse IRAC point spread function (PSF). We generated 24,000 artificial point sources for each image, uniformly distributed between 13 and 25 mags. Our artificial point sources were simply copies of the PSF for each image, which we generated by median combining unsaturated stars taken directly from each image. We added these sources to the original images ten at a time, ran Source Extractor again for each new image, and finally calculated the recovery rate as a function of magnitude for a given image and filter.

Figure 1 shows a histogram of the measured $50 \%$ point source completeness limits for each of our cluster images and control images at 3.6 (left) and $4.5 \mu \mathrm{m}$ (right). For comparison the vertical black line denotes the $50 \%$ completeness limit in each band from the SDWFS survey. We only want to fit for the cluster galaxy LF when the completeness of all galaxies in all clusters is at least $50 \%$. Therefore we limit our LF fitting procedure to the the brightest $50 \%$ point source completeness limit for all our clusters, which is 20.37 (19.60) mags in $3.6(4.5) \mu \mathrm{m}$. The $50 \%$ completeness limit for our cluster images is $\sim 0.75$ mags fainter than for the SDWFS images and $\sim 1$ mag brighter than our control images.

\section{OBSERVED LUMINOSITY FUNCTION}

\subsection{Optical-NIR Color Cut}

We use a simple color cut to remove stars and lowredshift galaxies from our catalogs and increase the signal-to-noise ratio of our high-redshift cluster galaxies. Our color cut is designed to include the blue cloud, as excluding part of it would induce a systematic bias in our measurement of the faint-end slope. We choose our color cut by using the Bruzual \& Charlot (2003) stellar models to create a model of a star-forming galaxy with a color on the blue side of the blue cloud, consistent with Lemaux et al. (2012). We then use this same model to estimate the color of the bluest star forming galaxies in our clusters, finding F775W-[3.6] $\geq 3.5$ and F775W-[4.5] $\geq$ 3.75. We use these values for our color cut, and note that our final results are not sensitive to our exact choice because our results change by less than our random errors for a wide range of color cuts $(2.5<\mathrm{F} 775 \mathrm{~W}-[3.6]<5)$.

Stellar contamination is a potential issue for our sample as our cluster and control fields are at different galactic latitudes. A [3.6]-[4.5] cut could effectively remove stars from our sample, but is not possible because there is little overlap between the 3.6 and $4.5 \mu \mathrm{m}$ imaging of our control regions. Limiting our sample to areas with 3.6 and $4.5 \mu \mathrm{m}$ imaging would remove about $75 \%$ of our control region. However, stellar contamination is effectively removed via our optical-NIR color cut. To verify that stellar contamination is not an issue for our results we calculate the expected colors for local stellar populations. To do this we download stellar isochrones using the CMD 2.3 software $^{8}$ which includes the latest stellar modeling details from a number of sources (Bonatto et al. 2004; Girardi et al. 2008; Marigo et al. 2008; Girardi et al. 2010). For a low metallicity $(Z=0.008)$ model, which is relevant to the Galactic halo, no star of any age has F775W-[3.6] $\gtrsim 4$. Solar metallicity stars have F775W-[3.6] 5, at the reddest. Therefore, only the tip of the RGB and AGB for old stars extend redder than the color cut. As such, only a small fraction of stars might remain after the cut, meaning that stellar contamination is not an issue. This is confirmed by the fact that our results do not change even when using color cuts as red as $\mathrm{F} 775 \mathrm{~W}-[4.5]=5$, which removes all stellar contamination. We additionally remove from our catalogs all objects with CLASS_STAR $>0.8$ in the ACS catalogs. We find that this has a negligible impact on our results, again showing that stellar contamination

\footnotetext{
${ }^{8}$ http://stev.oapd.inaf.it/cmd
} 


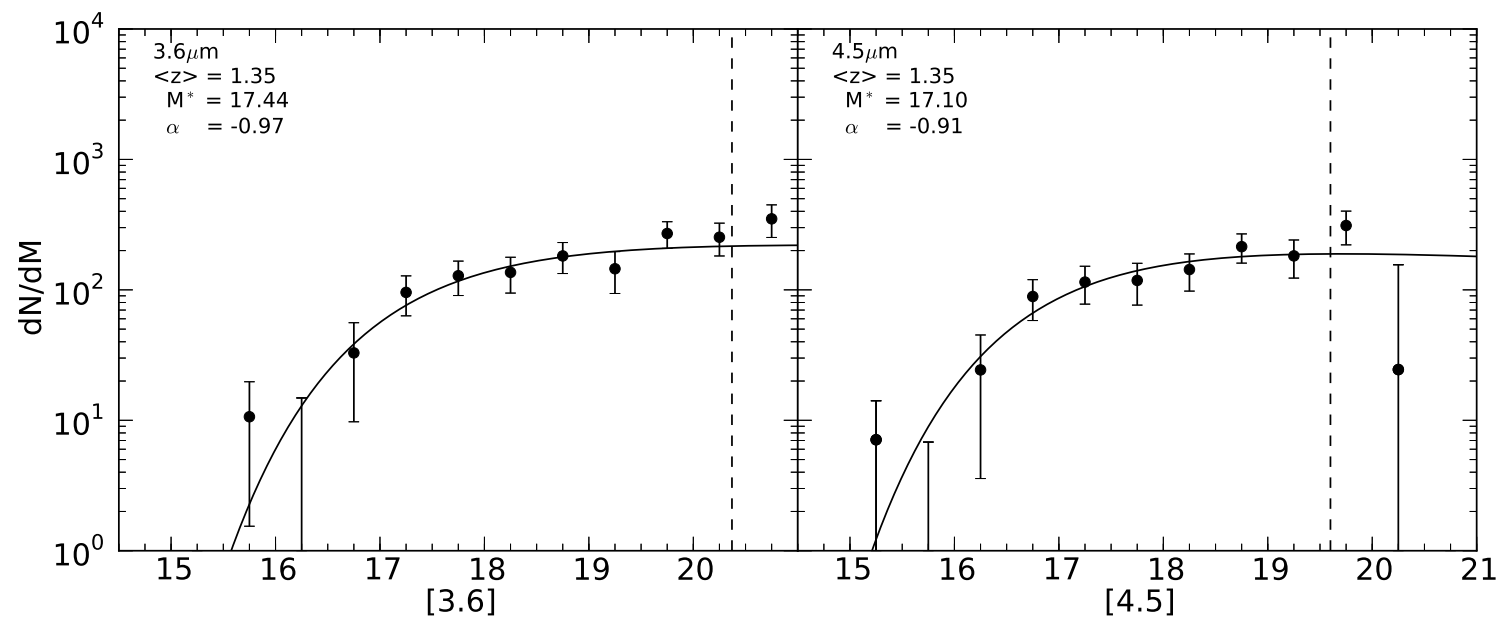

FIG. 2.- Binned and background-subtracted luminosity functions for 3.6 (left) and $4.5 \mu \mathrm{m}$ (right). Median cluster redshift and best fitting Schechter parameters are displayed in the top left. The solid curve shows the best fit. The dashed vertical line illustrates the magnitude limit for the fit, which is determined by the $50 \%$ completeness limit of the shallowest cluster image.

is not an issue for our sample.

\subsection{LF Fitting Procedure}

We design our methodology so that we can perform an unbinned fit to the cluster member LF, and so that the background subtraction is done in a way equivalent to a subtraction in observed space. We generate an individual cluster LF and control LF for every cluster. The cluster LF is simply composed of the galaxies in the cluster image which passed our various cuts (Section 3.1), are within $1.5 \mathrm{Mpc}$ of the cluster center, and are outside of the heavily blended cluster cores (typically $\sim 100 \mathrm{kpc}$ ). The latter restriction also removes the BCGs from our sample, which are known to not follow a Schechter distribution. For each cluster we use a Bruzual \& Charlot (2003) SPS model to calculate the $k$-correction and distance modulus correction needed to move a passively evolving galaxy $\left(z_{f}=3\right.$, Chabrier IMF, solar metallicity) from the cluster redshift to the median redshift of our cluster sample $(z=1.35)$. We then apply this $k$-correction and distance modulus correction to all galaxies in the cluster LF. Next we build a control LF for each cluster in a similar fashion. We select all galaxies in the control images which pass our cuts, weight them according to the relative area of the cluster and field images, and apply the exact same $k$-correction and distance modulus correction that we applied to the cluster LF to all the galaxies in the control LF. We do this so that the same transformation has been applied in the same way to the cluster and control galaxies, and therefore when we subtract the control LF from the cluster LF the subtraction is effectively done in observed space.

This procedure gives us an unbinned cluster and control LF for each cluster. We then combine the individual cluster and control LFs into a composite cluster and composite control LF, which we use to measure the LF of cluster members. We parameterize the luminosity function of cluster members as a Schechter (1976) luminosity function and measure the best fitting Schechter parameters with maximum likelihood fitting, similar to the procedure used in Mancone et al. (2010). This procedure requires an analytical representation for the contribution from the control region so we bin our composite control

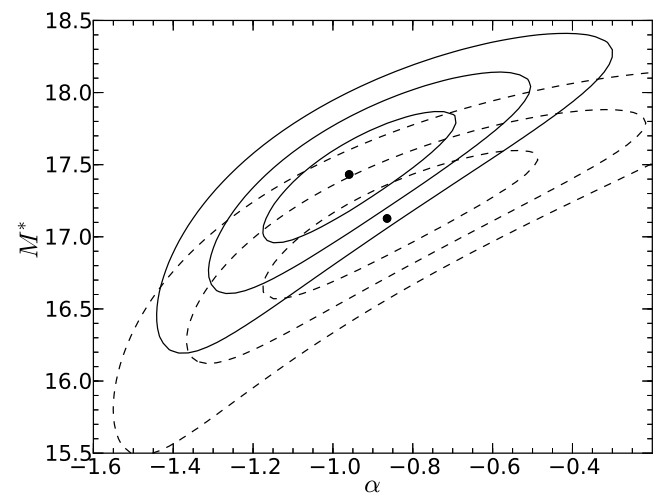

FIG. 3.- Confidence regions for the Schechter fits to our $3.6 \mu \mathrm{m}$ (filled) and $4.5 \mu \mathrm{m}$ (dashed) cluster LFs. Contours represent the 1,2 , and $3 \sigma$ confidence regions in $\alpha$ vs $M^{*}$ space. Filled circles denote the best fit Schechter parameters

LF by magnitude, correct for photometric incompleteness, and fit a third order polynomial to it in log space. We then use maximum likelihood fitting to fit the sum of a Schechter luminosity function (the cluster member LF) and the fitted composite control LF to the composite cluster LF. We use a downhill simplex algorithm (Press et al. 2007) to maximize the likelihood as a function of $\Phi^{*}, m^{*}$, and $\alpha$, and fit all galaxies brighter than the $50 \%$ completeness limit for the clusters (Section 2.3).

\subsection{Results}

Figure 2 shows the control-subtracted cluster LF and the Schechter fit to the cluster member LF for $3.6 \mu \mathrm{m}$ (left) and $4.5 \mu \mathrm{m}$ (right). Maximum likelihood fitting gives a fit to the LF without binning, but for plotting purposes we show the binned and control-subtracted cluster LF in Figure 2, which is the binned difference between the composite cluster LF and the composite control LF. In each panel the solid curve shows the best fit while the dashed vertical line illustrates the magnitude limit used for the fit. Figure 3 shows the 1, 2, and $3 \sigma$ contours in $M^{*}$ vs. $\alpha$ space derived from our measured likelihoods. We also report the binned LF values in Table 3, although we note that our fit was to the unbinned data. 
TABLE 2

Best Fitting Schechter Parameters

\begin{tabular}{cccccc}
\hline \hline$\langle z\rangle$ & \# Clusters & $M_{3.6 \mu \mathrm{m}}^{*}$ & $\alpha_{3.6 \mu \mathrm{m}}$ & $M_{4.5 \mu \mathrm{m}}^{*}$ & $\alpha_{4.5 \mu \mathrm{m}}$ \\
& & & & \\
\hline 1.35 & 7 & $17.44 \pm 0.30$ & $-0.97 \pm 0.14$ & $17.10 \pm 0.43$ & $-0.91 \pm 0.28$ \\
\hline
\end{tabular}

TABLE 3

BINNED LFS

\begin{tabular}{ccc}
\hline \hline Mag & $\#[3.6]$ & $\#[4.5]$ \\
\hline 15.25 & $-1.32 \pm 0.50$ & $7.09 \pm 7.02$ \\
15.75 & $10.64 \pm 9.10$ & $-3.31 \pm 10.12$ \\
16.25 & $0.87 \pm 13.97$ & $24.34 \pm 20.76$ \\
16.75 & $32.86 \pm 23.12$ & $88.94 \pm 30.76$ \\
17.25 & $95.61 \pm 32.39$ & $114.71 \pm 37.00$ \\
17.75 & $128.29 \pm 37.82$ & $118.11 \pm 41.72$ \\
18.25 & $136.13 \pm 41.68$ & $143.18 \pm 45.37$ \\
18.75 & $181.98 \pm 48.57$ & $214.39 \pm 54.09$ \\
19.25 & $145.11 \pm 51.32$ & $182.13 \pm 59.06$ \\
19.75 & $270.40 \pm 62.50$ & $\ldots$ \\
20.25 & $253.25 \pm 71.37$ & $\ldots$ \\
& & \\
\hline
\end{tabular}

We also measure uncertainties using bootstrap resampling, as such an error estimate is more sensitive to systematic uncertainties caused by cluster-to-cluster variations. We generate realizations of the LF by randomly selecting seven clusters from our sample and repeating our LF fitting procedure with the new cluster sample. The cluster selection is done with replacement, which means that an individual cluster can be selected more than once when generating a new cluster sample. This allows us to probe any systematic uncertainty caused by cluster-to-cluster variation, as this process effectively applies random weights to the clusters while fitting. We perform 100 realizations of the cluster LF and take the standard deviation of the fitted $M^{*}$ and $\alpha$ parameters as our random uncertainties. Our best fitting Schechter parameters and bootstrap errors are listed in Table 2 . We note that our measured bootstrap uncertainties agree well with the contours in Figure 3.

The relatively small sizes of the GOODS fields means that cosmic variance in our control fields could be an additional source of systematic uncertainty. To verify that cosmic variance is not strongly biasing our results we redo our fit but use just one of the GOODS fields for our control sample, and then redo it again using the other. In each case the best fitting value of $\alpha$ changes by $\sim 0.1$ in both filters, which is smaller than our measured errors. Therefore, cosmic variance is unlikely to be a dominant source of uncertainty in our results.

\section{DISCUSSION}

\subsection{High-Redshift Comparison}

For a basic consistency check we compare to our results from Mancone et al. (2010). In Mancone et al. (2010) we measured $M_{3.6 \mu \mathrm{m}}^{*}$ and $M_{4.5 \mu \mathrm{m}}^{*}$ out to $z=1.8$ using the slightly shallower SDWFS data and a statistical background subtraction. As such the methodology is very similar, the filters are the same, and the seven clusters studied herein were also included in Mancone et al. (2010). Due to our shallower data in Mancone et al. (2010) we fixed $\alpha$ and reported fitted $M^{*}$ values for $\alpha=-0.6,-0.8$, and -1.0 in redshift bins from $z=0.3$ to $z=2.0$. The median redshift of the clusters in this study $(z=1.35)$ fall directly between the $z=1.24$ and $z=1.46$ bins from Mancone et al. (2010). Therefore we compare our fitted $M^{*}$ values to the average of the $M^{*}$ values for the $z=1.24$ and 1.46 bins with $\alpha=-1.0$, which gives $M_{3.6 \mu \mathrm{m}}^{*}=17.42 \pm 0.1$ and $M_{4.5 \mu \mathrm{m}}^{*}=16.92 \pm 0.1$, in good agreement with the values of $M^{*}$ measured herein.

We note that our random errors for $M^{*}$ in this paper are larger than the quoted errors in Mancone et al. (2010). This is a simple result of number statistics. While we only have seven clusters in this study, we had 25 (22) clusters in our $z=1.24$ (1.46) bins in Mancone et al. (2010). This leads to a larger random uncertainty in $M^{*}$ for this current work, although we have lower systematic uncertainty for $M^{*}$ in this paper because the requirement of fixing $\alpha$ in Mancone et al. (2010) introduced a systematic uncertainty of $\sim 0.2$ mags into $M^{*}$.

We also compare to Strazzullo et al. (2010) who measure the $H$-band LF of a $z=1.39$ galaxy cluster to $M^{*}+4$. They find $\alpha_{H}=-1.2_{-0.15}^{+0.2}$, also consistent with our results. While there is a difference in passband between our studies, both trace rest-frame wavelengths redward of the $4000 \AA$ break so we expect the difference in passband to have a minimal affect on our fitted values of $\alpha$.

Recent studies have found a deficit of low-luminosity red-sequence galaxies in high-redshift clusters (De Lucia et al. 2004; Rudnick et al. 2009; Lemaux et al. 2012). At face value this seems in contradiction with the flat $\alpha$ values found in this study as well as Strazzullo et al. (2010). However, neither our results nor the results from Strazzullo et al. (2010) are limited to red-sequence galaxies, and therefore this apparent difference can simply be a sign that low-luminosity galaxies are in place in the cluster environment at these redshifts but have not yet finished transitioning onto the red sequence, as was suggested in Lemaux et al. (2012).

\subsection{Low-Redshift Comparison}

We compare our results to low-redshift cluster LFs to assess the evolution of $\alpha$ over a substantial fraction $(\sim 9$ Gyr) of cosmic history. To accomplish this we have compiled a list of $\alpha$ measurements from the literature for a variety of clusters or cluster samples at different redshifts, which we summarize in Table 4 . We note that de Propris et al. (1998), Andreon (2001), and De Propris \& Christlein (2009) did not present a formal error for $\alpha$ but did plot confidence regions for their fit, so we estimated the error on $\alpha$ from the plots of their confidence regions. Specifically, we derived the error from the full range of values covered by their $1 \sigma$ confidence contours. We split Table 4 up into two sections: studies which trace the restframe optical and studies which trace the rest-frame NIR 
TABLE 4

$\alpha$ Values From the Literature

\begin{tabular}{cccccc}
\hline \hline Reference & Cluster & \# Clusters & Band & $\langle z\rangle$ & $\alpha$ \\
& & & & & \\
& & & & & \\
\hline & Rest-Frame NIR & & & \\
\hline de Propris et al. (1998) & Coma & 1 & $\mathrm{H}$ & 0.023 & $-0.78 \pm 0.3^{\mathrm{b}}$ \\
Andreon (2001) & $\mathrm{AC} 118$ & 1 & $\mathrm{Ks}$ & 0.3 & $-1.18 \pm 0.15^{\mathrm{b}}$ \\
Lin et al. (2004) & $\ldots$ & 93 & $\mathrm{Ks}$ & 0.043 & $-0.84 \pm 0.02$ \\
Jenkins et al. (2007) & Coma & 1 & $3.6 \mu \mathrm{m}$ & 0.023 & $-1.25 \pm 0.05$ \\
Muzzin et al. (2007) & $\ldots$ & 15 & $\mathrm{~K}$ & 0.296 & $-0.84 \pm 0.08$ \\
Skelton et al. (2009) & Norma & 1 & $\mathrm{Ks}$ & 0.016 & $-1.26 \pm 0.1$ \\
De Propris \& Christlein (2009) & $\ldots$ & 10 & $\mathrm{~K}$ & 0.07 & $-0.98 \pm 0.2^{\mathrm{b}}$ \\
Mancone et al. (2010) & $\ldots$ & 35 & $3.6 \mu \mathrm{m}$ & 0.37 & $-0.60 \pm 0.2$ \\
This Work & $\ldots$ & 7 & $3.6 \mu \mathrm{m}$ & 1.35 & $-0.97 \pm 0.14$ \\
This Work & $\ldots$ & $7.5 \mu \mathrm{m}$ & 1.35 & $-0.91 \pm 0.28$ \\
\hline & Rest-Frame Optical & & & \\
\hline Mobasher et al. (2003) & Coma & 1 & $\mathrm{R}$ & 0.023 & $-1.18_{-0.02}^{+0.04}$ \\
De Propris et al. (2003) & $\ldots$ & 60 & $\mathrm{Bj}$ & $<0.11$ & $-1.28 \pm 0.03$ \\
Chiboucas \& Mateo (2006) & Centaurus & 1 & $\mathrm{~V}$ & 0.0114 & $-1.4_{-0.1}^{+0.1}$ \\
Strazzullo et al. (2010) & XMMU J2235-2557 & 1 & $\mathrm{H}$ & 1.39 & $-1.2_{-0.15}^{+0.2}$ \\
\hline & & & & & \\
\hline
\end{tabular}

${ }^{\mathrm{a}} \mathrm{A}$ cluster name is given only when a single cluster is studied in the given paper.

${ }^{b}$ Formal errors were not given but were estimated from plots of confidence regions.

(such as this work). Star formation can be an important contributor to the rest-frame optical LF, and as such $\alpha$ is not necessarily directly comparable between the two sets of studies.

The results in Table 4 are presented graphically in Figure 4. In this Figure the fitted $\alpha$ values and errors are plotted for all the rest-frame NIR results in Table 4. There is substantial study-to-study scatter at low redshift, and large error bars at high redshift, but Figure 4 shows no obvious evidence for evolution in $\alpha$ from $z=0$ to $z \sim 1.4$, representing nearly $70 \%$ of cosmic history.

Past work on cluster LFs have primarily characterized the evolution of $M^{*}$, and shallow imaging has required assuming a value for $\alpha$ and fixing it as a function of redshift (see, e.g., Muzzin et al. 2008 and Mancone et al. 2010). Fixing $\alpha$ has been a potential source of systematic uncertainty, as the strong coupling between $M^{*}$ and $\alpha$ means that if $\alpha$ is improperly held fixed then the fitted values of $M^{*}$ will also be wrong. This can be particularly important for studies of the evolution of $M^{*}$ because if $\alpha$ is evolving but assumed to be fixed then this false assumption can create spurious evolution in $M^{*}$. This potential source of systematic uncertainty was discussed in detail in Mancone et al. (2010) because we found that for $z \gtrsim 1.3$ the fitted values of $M^{*}$ to the cluster LF deviated strongly from passive evolution. We concluded that while evolution in $\alpha$ could contribute to the measured deviations from passive evolution, it was unlikely to be the underlying cause because the direction of the deviation would require $\alpha$ to become steeper at higher redshift. Having now measured $\alpha$ at high redshift we can conclude that evolution in $\alpha$ was not the cause of our observed deviations from passive evolution as $\alpha$ does not evolve significantly with redshift out to $z \sim 1.4$.

\subsection{Comparison to the Field LF}

We find that at high redshift the faint-end slope of the cluster LF matches that of the field. Saracco et al. (2006)

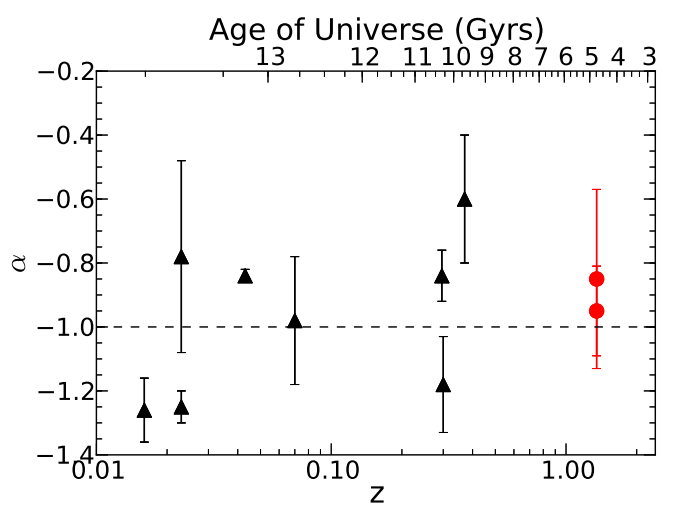

FIG. 4.- Best-fitting values of $\alpha$ to the cluster luminosity function versus redshift. Data points come from the rest-frame NIR studies from Table 4 and include a variety of literature results. The results from this work are shown as red circles while previous results are shown as black triangles. The dashed horizontal line denotes $\alpha=-1.0$, corresponding to a flat LF for faint galaxies.

measured $\alpha$ in the rest-frame $J$-band for field galaxies, finding $\alpha=-0.94_{-0.15}^{+0.16}$ in a redshift bin centered at $z \sim$ 1.2. Cirasuolo et al. (2007) found $\alpha=-0.92 \pm 0.18$ for galaxies with $1.25<z<1.5$ in the rest-frame $K$-band, again in good agreement with the faint-end slope of the cluster LF found herein. Moreover, work in the field (Saracco et al. 2006; Cirasuolo et al. 2007; Stefanon \& Marchesini 2011) has also found that the faint end of the LF for field galaxies is consistent with being constant out to the highest redshifts studied. For example, Kochanek et al. (2001) find $\alpha=-1.09 \pm 0.06$ for 2MASS galaxies at low redshift, while Stefanon \& Marchesini (2011) find that the faint-end slope of their rest-frame $J$-band LF is consistent with flat from $1.5<z<3.5$. They compare their results to lower redshift studies, finding no evidence for evolution in $\alpha$ out to $z=3.5$ with a mean value of $\alpha=-1.05 \pm 0.03$. This is all consistent with our finding that $\alpha$ does not substantially evolve but is consistent 
with flat from $z=0$ to $z \sim 1.4$ for cluster galaxies.

\subsection{Implications for Galaxy Formation}

As discussed above, we find no statistically significant evidence for evolution in the faint-end slope of the NIR luminosity function out to $z \sim 1$.4. A lack of evolution in $\alpha$ combined with a lack of evolution in $M^{*}$ (Mancone et al. 2010) means that both faint and bright galaxies are largely in place at high redshift. This places a strong constraint on the luminosity evolution of cluster galaxies at $z \lesssim 1.4$. Either little evolution is happening at lower redshifts, or the processes responsible for LF evolution have no net impact on the cluster population.

To understand the implications of this for galaxy evolution, we must understand how the various process that cause cluster galaxy evolution would affect the luminosity evolution of the cluster galaxy population. The fact that the NIR traces old stellar populations means that our results are most sensitive to processes which would cause evolution in the stellar mass of cluster galaxies. Any process which affects low and high stellar mass cluster galaxies equally will lead to evolution in $M^{*}$. Conversely, any process which leads to differential evolution between galaxies with low and high stellar masses will lead to evolution in $\alpha$.

The two primary processes by which galaxies can grow their stellar masses over time are star formation and mergers. The downsizing paradigm (see Fontanot et al. 2009 and references therein) suggests that star formation will be preferentially found in lower mass galaxies at low redshift. Such a mass dependence for galaxy star formation histories will necessarily imply evolution in the faint-end slope of the LF. The amplitude of this effect however depends upon the total amount of ongoing star formation, which will vary between clusters and may depend upon the total mass of the host cluster halo. There is evidence that substantial star formation is still ongoing in our cluster sample (Snyder et al. 2012), as well as other clusters at similar redshifts (Hilton et al. 2010; Tran et al. 2010; Fassbender et al. 2011). In contrast, Muzzin et al. (2012) find that star formation has already been strongly quenched in their cluster at $z=1.2$.

Mergers can also build up the stellar mass of cluster galaxies, although mergers are expected to be suppressed in the cluster environment due to the high relative velocities of cluster galaxies (Alonso et al. 2012). Recent theoretical studies (Murante et al. 2007; Conroy et al. 2007; Puchwein et al. 2010) suggest that for massive galaxies growth by mergers becomes very inefficient (but see Rudnick et al. 2012), and it is possible that mergers can yield a steepening of the faint-end slope if this efficiency is strongly mass-dependent.

In contrast gravitational interactions such as galaxygalaxy interactions, interactions of a galaxy with the cluster potential, galaxy harassment, and the dissolution of cluster galaxies, can all strip mass away from lowmass galaxies in particular (Moore et al. 1996; Boselli \& Gavazzi 2006; Murante et al. 2007) and therefore cause $\alpha$ to grow flatter or turn over with time.

Another process for consideration is the infall of new galaxies into the cluster, the effect of which depends on the shape of the LF for the infalling galaxy population. Since we find that the cluster and field galaxy populations have a similar faint end slope (Section 4.3), we ex- pect that the infall of new galaxies into the cluster will primarily act to mitigate any potential evolution of the cluster LF by driving the cluster LF back towards a flat faint-end slope. Instead, the infall of new galaxies will lead to an increase in $\Phi^{*}$, the normalization of the LF, which we have not constrained

Finally, a mass-dependent galactic initial mass function (IMF) can cause the shape of the LF to change relative to the underlying stellar mass function. This is because the rate of luminosity evolution for a stellar population depends sensitively on the IMF (Conroy et al. 2009). Recent work (Cappellari et al. 2012) has suggested that the IMF does indeed depend on galaxy mass, such that lower mass galaxies have a flatter IMF (i.e., a higher fraction of high-mass stars). A flatter IMF leads to a faster fading of the underlying stellar population (Conroy et al. 2009) and therefore, if true, the results of Cappellari et al. (2012) suggest that low-mass galaxies should fade faster than high mass galaxies when the stellar masses of both remains fixed. This will cause $\alpha$ to grow flatter or turn over with time, effectively acting against processes which build up the stellar mass of galaxies but without impacting the underlying mass function.

Clearly, there are many processes which could potentially cause evolution of the NIR cluster LF at $z<1.5$. Therefore, the lack of evolution in $\alpha$ observed herein, combined with the lack of evolution in $M^{*}$ observed over a similar redshift range (Mancone et al. 2010), places an important constraint on these processes. In net, they cannot cause any large evolution in the shape of the NIR cluster LF. This could be because both low mass and high mass cluster galaxies are largely assembled at high redshift, or because the differing effects of these processes causes little evolution in net. The latter might imply an uncomfortable degree of fine tuning. In general though, the ability of infalling galaxies to dilute any evolution in the cluster LF allows for more flexibility in the strength of other processes.

\section{CONCLUSIONS}

We measure the 3.6 and $4.5 \mu \mathrm{m}$ luminosity functions of seven galaxy clusters at $1<z<1.5$, specifically investigating the shape of the $\mathrm{LF}$ for faint galaxies. We find the LFs to be well-fit by Schechter distributions with faint-end slopes of $\alpha_{3.6 \mu \mathrm{m}}=-0.97 \pm 0.14$ and $\alpha_{4.5 \mu \mathrm{m}}=-0.91 \pm 0.28$, both consistent with having flat faint-end slopes within $1 \sigma$. Our primary conclusions are summarized here:

1. We compare to studies of the NIR LF of lowredshift clusters and find no statistically significant evidence for evolution of the faint-end slope of the cluster LF. Therefore we conclude that the faint end of the cluster LF has not evolved significantly over $70 \%$ of cosmic history.

2. Having measured a non-evolving faint-end slope we have removed one source of systematic uncertainty from studies of the evolution of $M^{*}$ as a function of redshift. This is particularly relevant for our recent detection of deviations from passive evolution at high redshift (Mancone et al. 2010). Shallow imaging in Mancone et al. (2010) necessitated fix- 
ing $\alpha$, which could have lead to spurious evolution in $M^{*}$ if $\alpha$ was evolving.

3. We compare to the faint end slope for field galaxies at similar redshifts and find good agreement. Field studies (Saracco et al. 2006; Cirasuolo et al. 2007; Stefanon \& Marchesini 2011) find a faint-end slope consistent with flat at high redshift, and the most recent results (Stefanon \& Marchesini 2011) find no evidence for evolution out to $z=3.5$.

4. Given recent studies (Muzzin et al. 2008; Mancone et al. 2010) which have found that the evolution of the bright end of the cluster LF is consistent with passive evolution out to $z \sim 1.3$, we conclude that the shape of the cluster LF has been in place and evolved little since $z \sim 1.3$. This could suggest that low-mass galaxies are largely assembled at high redshift. Conversely, it could simply mean that the many processes which cause evolution of the cluster galaxy population have no net impact on the mass and luminosity function of cluster galaxies.

This work is based on observations made with the Spitzer Space Telescope, which is operated by the Jet Propulsion Laboratory, California Institute of Technology under a contract with NASA. The observations are associated with programs P78 and P30950. This work is based on observations made with the NASA/ESA Hubble Space Telescope, obtained from the Data Archive at the Space Telescope Science Institute, which is operated by the Association of Universities for Research in Astronomy, Inc., under NASA contract NAS 5-26555. The observations are associated with program GO-10496. CLM and AHG acknowledge support for this work from the National Science Foundation under grant AST-00708490. AHG, MB, and SAS also acknowledge support from NASA through grant HST-GO-10496.

\section{REFERENCES}

Alonso, S., Mesa, V., Padilla, N., \& Lambas, D. G. 2012, A\&A, $539, \mathrm{~A} 46$

Andreon, S. 2001, ApJ, 547, 623

-. 2006, A\&A, 448, 447

Andreon, S., Trinchieri, G., \& Pizzolato, F. 2011, MNRAS, 412, 2391

Ashby, M. L. N., Stern, D., Brodwin, M., Griffith, R., Eisenhardt, P., Kozłowski, S., Kochanek, C. S., Bock, J. J., Borys, C., Brand, K., Brown, M. J. I., Cool, R., Cooray, A., Croft, S., Dey, A., Eisenstein, D., Gonzalez, A. H., Gorjian, V., Grogin, N. A. Ivison, R. J., Jacob, J., Jannuzi, B. T., Mainzer, A., Moustakas, L. A., Röttgering, H. J. A., Seymour, N., Smith, H. A., Stanford, S. A., Stauffer, J. R., Sullivan, I., van Breugel, W., Willner, S. P., \& Wright, E. L. 2009, ApJ, 701, 428

Bertin, E. \& Arnouts, S. 1996, A\&AS, 117, 393

Bonatto, C., Bica, E., \& Girardi, L. 2004, A\&A, 415, 571

Boselli, A. \& Gavazzi, G. 2006, PASP, 118, 517

Brodwin, M., Brown, M. J. I., Ashby, M. L. N., Bian, C., Brand, K., Dey, A., Eisenhardt, P. R., Eisenstein, D. J., Gonzalez, A. H., Huang, J.-S., Jannuzi, B. T., Kochanek, C. S., McKenzie, E., Murray, S. S., Pahre, M. A., Smith, H. A., Soifer, B. T., Stanford, S. A., Stern, D., \& Elston, R. J. 2006, ApJ, 651, 791

Brodwin, M., Stern, D., Vikhlinin, A., Stanford, S. A., Gonzalez, A. H., Eisenhardt, P.' R., Ashby, M. L. N., Bautz, M., Dey, A., Forman, W. R., Gettings, D., Hickox, R. C., Jannuzi, B. T., Jones, C., Mancone, C., Miller, E. D., Moustakas, L. A., Ruel, J., Snyder, G., \& Zeimann, G. 2011, ApJ, 732, 33

Bruzual, G. \& Charlot, S. 2003, MNRAS, 344, 1000

Cappellari, M., McDermid, R. M., Alatalo, K., Blitz, L., Bois, M.,

Bournaud, F., Bureau, M., Crocker, A. F., Davies, R. L., Davis,

T. A., de Zeeuw, P. T., Duc, P.-A., Emsellem, E., Khochfar, S., Krajnovic, D., Kuntschner, H., Lablanche, P.-Y., Morganti, R., Naab, T., Oosterloo, T., Sarzi, M., Scott, N., Serra, P., Weijmans, A.-M., \& Young, L. M. 2012, ArXiv e-prints

Chen, Y.-M., Wild, V., Kauffmann, G., Blaizot, J., Davis, M., Noeske, K., Wang, J.-M., \& Willmer, C. 2009, MNRAS, 393, 406

Chiboucas, K. \& Mateo, M. 2006, AJ, 132, 347

Cirasuolo, M., McLure, R. J., Dunlop, J. S., Almaini, O., Foucaud, S., Smail, I., Sekiguchi, K., Simpson, C., Eales, S., Dye, S., Watson, M. G., Page, M. J., \& Hirst, P. 2007, MNRAS, 380 585

Conroy, C., Gunn, J. E. \& White, M. 2009, ApJ, 699, 486

Conroy, C., Wechsler, R. H., \& Kravtsov, A. V. 2007, ApJ, 668, 826

Cowie, L. L., Songaila, A., Hu, E. M., \& Cohen, J. G. 1996, AJ, 112,839

De Lucia, G., Poggianti, B. M., Aragón-Salamanca, A., Clowe, D., Halliday, C., Jablonka, P., Milvang-Jensen, B., Pelló, R., Poirier S., Rudnick, G., Saglia, R., Simard, L., \& White, S. D. M. 2004 ApJ, 610, L77

De Propris, R. \& Christlein, D. 2009, Astronomische Nachrichten, 330, 943
De Propris, R., Colless, M., Driver, S. P., Couch, W., Peacock, J. A., Baldry, I. K., Baugh, C. M., Bland-Hawthorn, J., , Cannon, R., Cole, S., Collins, C., Cross, N., Dalton, G. B., Efstathiou, G., Ellis, R. S., Frenk, C. S., Glazebrook, K., Hawkins, E., Jackson, C., Lahav, O., Lewis, I., Lumsden, S., Maddox, S., Madgwick, D. S., Norberg, P., Percival, W., Peterson, B., Sutherland, W., \& Taylor, K. 2003, MNRAS, 342, 725

de Propris, R., Eisenhardt, P. R., Stanford, S. A., \& Dickinson, M. 1998, ApJ, 503, L45

De Propris, R., Stanford, S. A., Eisenhardt, P. R., Holden, B. P., \& Rosati, P. 2007, AJ, 133, 2209

Desai, V., Dalcanton, J. J., Aragón-Salamanca, A., Jablonka, P., Poggianti, B., Gogarten, S. M., Simard, L., Milvang-Jensen, B., Rudnick, G., Zaritsky, D., Clowe, D., Halliday, C., Pelló, R., Saglia, R., \& White, S. 2007, ApJ, 660, 1151

Dickinson, M., Giavalisco, M., \& GOODS Team. 2003, in The Mass of Galaxies at Low and High Redshift, ed. R. Bender \& A. Renzini, 324

Dressler, A., Oemler, Jr., A., Couch, W. J., Smail, I., Ellis, R. S., Barger, A., Butcher, H., Poggianti, B. M., \& Sharples, R. M. 1997, ApJ, 490, 577

Eisenhardt, P. R. M., Brodwin, M., Gonzalez, A. H., Stanford, S. A., Stern, D., Barmby, P., Brown, M. J. I., Dawson, K., Dey, A., Doi, M., Galametz, A., Jannuzi, B. T., Kochanek, C. S., Meyers, J., Morokuma, T., \& Moustakas, L. A. 2008, ApJ, 684, 905

Elston, R. J., Gonzalez, A. H., McKenzie, E., Brodwin, M., Brown, M. J. I., Cardona, G., Dey, A., Dickinson, M., Eisenhardt, P. R., Jannuzi, B. T., Lin, Y.-T., Mohr, J. J., Raines, S. N., Stanford, S. A., \& Stern, D. 2006, ApJ, 639, 816

Fassbender, R., Nastasi, A., Böhringer, H., Šuhada, R., Santos, J. S., Rosati, P., Pierini, D., Mühlegger, M., Quintana, H., Schwope, A. D., Lamer, G., de Hoon, A., Kohnert, J., Pratt, G. W., \& Mohr, J. J. 2011, A\&A, 527, L10

Fontanot, F., De Lucia, G., Monaco, P., Somerville, R. S., \& Santini, P. 2009, MNRAS, 397, 1776

Girardi, L., Dalcanton, J., Williams, B., de Jong, R., Gallart, C., Monelli, M., Groenewegen, M. A. T., Holtzman, J. A., Olsen, K. A. G., Seth, A. C., Weisz, D. R., \& the ANGST/ANGGRR Collaboration. 2008, PASP, 120, 583

Girardi, L., Williams, B. F., Gilbert, K. M., Rosenfield, P., Dalcanton, J. J., Marigo, P., Boyer, M. L., Dolphin, A., Weisz, D. R., Melbourne, J., Olsen, K. A. G., Seth, A. C., \& Skillman, E. 2010, ApJ, 724, 1030

Hilton, M., Lloyd-Davies, E., Stanford, S. A., Stott, J. P., Collins, C. A., Romer, A. K., Hosmer, M., Hoyle, B., Kay, S. T., Liddle, A. R., Mehrtens, N., Miller, C. J., Sahlén, M., \& Viana, P. T. P. 2010, ApJ, 718, 133

Jee, M. J., Dawson, K. S., Hoekstra, H., Perlmutter, S., Rosati, P., Brodwin, M., Suzuki, N., Koester, B., Postman, M., Lubin, L., Meyers, J., Stanford, S. A., Barbary, K., Barrientos, F., Eisenhardt, P., Ford, H. C., Gilbank, D. G., Gladders, M. D., Gonzalez, A., Harris, D. W., Huang, X., Lidman, C., Rykoff,' E. S., Rubin, D., \& Spadafora, A. L. 2011, ApJ, 737, 59

Jenkins, L. P., Hornschemeier, A. E., Mobasher, B., Alexander, D. M., \& Bauer, F. E. 2007, ApJ, 666, 846 
Kochanek, C. S., Pahre, M. A., Falco, E. E., Huchra, J. P., Mader, J., Jarrett, T. H., Chester, T., Cutri, R., \& Schneider, S. E. 2001, ApJ, 560, 566

Komatsu, E., Smith, K. M., Dunkley, J., Bennett, C. L., Gold, B., Hinshaw, G., Jarosik, N., Larson, D., Nolta, M. R., Page, L., Spergel, D. N., Halpern, M., Hill, R. S., Kogut, A., Limon, M., Meyer, S. S., Odegard, N., Tucker, G. S., Weiland, J. L., Wollack, E., \& Wright, E. L. 2011, ApJS, 192, 18

Lemaux, B. C., Gal, R. R., Lubin, L. M., Kocevski, D. D. Fassnacht, C. D., McGrath, E. J., Squires, G. K., Surace, J. A., \& Lacy, M. 2012, ApJ, 745, 106

Lin, Y.-T., Mohr, J. J., \& Stanford, S. A. 2004, ApJ, 610, 745

Lu, T., Gilbank, D. G., Balogh, M. L., \& Bognat, A. 2009, MNRAS, 399, 1858

Mancone, C. L. \& Gonzalez, A. H. 2012, PASP, 124, 606

Mancone, C. L. Gonzalez, A. H., Brodwin, M., Stanford, S. A. Eisenhardt, P. R. M., Stern, D., \& Jones, C. 2010, ApJ, 720, 284

Marigo, P., Girardi, L., Bressan, A., Groenewegen, M. A. T., Silva, L., \& Granato, G. L. 2008, A\&A, 482, 883

Mobasher, B., Colless, M., Carter, D., Poggianti, B. M., Bridges, T. J., Kranz, K., Komiyama, Y., Kashikawa, N., Yagi, M., \& Okamura, S. 2003, ApJ, 587, 605

Mobasher, B., Dahlen, T., Hopkins, A., Scoville, N. Z., Capak, P., Rich, R. M., Sanders, D. B., Schinnerer, E., Ilbert, O., Salvato, M., \& Sheth, K. 2009, ApJ, 690, 1074

Moore, B., Katz, N., Lake, G., Dressler, A., \& Oemler, A. 1996, Nature, 379, 613

Murante, G., Giovalli, M., Gerhard, O., Arnaboldi, M., Borgani, S., \& Dolag, K. 2007, MNRAS, 377, 2

Muzzin, A., Wilson, G., Lacy, M., Yee, H. K. C., \& Stanford, S. A. 2008, ApJ, 686, 966

Muzzin, A., Wilson, G., Yee, H. K. C., Gilbank, D., Hoekstra, H., Demarco, R., Balogh, M., van Dokkum, P., Franx, M., Ellingson, E., Hicks, A., Nantais, J., Noble, A., Lacy, M., Lidman, C., Rettura, A., Surace, J., \& Webb, T. 2012, ApJ, 746, 188

Muzzin, A., Yee, H. K. C., Hall, P. B., Ellingson, E., \& Lin, H. 2007, ApJ, 659, 1106

Panter, B., Jimenez, R., Heavens, A. F., \& Charlot, S. 2007, MNRAS, 378, 1550

Press, W., Teukolsky, S., Vetterling, W., \& Flannery, B. 2007, Numerical Recipes 3rd Edition: The Art of Scientific Computing, 3rd edn. (Cambridge University Press)

Puchwein, E., Springel, V., Sijacki, D., \& Dolag, K. 2010, MNRAS, 406, 936

Reach, W. T., Megeath, S. T., Cohen, M., Hora, J., Carey, S., Surace, J., Willner, S. P., Barmby, P., Wilson, G., Glaccum, W. Lowrance, P., Marengo, M., \& Fazio, G. G. 2005, PASP, 117, 978

Rudnick, G., von der Linden, A., Pelló, R., Aragón-Salamanca, A., Marchesini, D., Clowe, D., De Lucia, G., Halliday, C., Jablonka, P., Milvang-Jensen, B., Poggianti, B., Saglia, R., Simard, L., White, S., \& Zaritsky, D. 2009, ApJ, 700, 1559
Rudnick, G. H., Tran, K.-V., Papovich, C., Momcheva, I., \& Willmer, C. 2012, ApJ, 755, 14

Saracco, P., Fiano, A., Chincarini, G., Vanzella, E., Longhetti, M., Cristiani, S., Fontana, A., Giallongo, E., \& Nonino, M. 2006, MNRAS, 367, 349

Schechter, P. 1976, ApJ, 203, 297

Skelton, R. E., Woudt, P. A., \& Kraan-Korteweg, R. C. 2009, MNRAS, 396, 2367

Snyder, G. F., Brodwin, M., Mancone, C. M., Zeimann, G. R., Stanford, S. A., Gonzalez, A. H., Stern, D., Eisenhardt, P. R. M., Brown, M. J. I., Dey, A., Jannuzi, B., \& Perlmutter, S. 2012, ApJ, 756, 114

Stanford, S. A., Eisenhardt, P. R., Brodwin, M., Gonzalez, A. H., Stern, D., Jannuzi, B. T., Dey, A., Brown, M. J. I., McKenzie, E., \& Elston, R. 2005, ApJ, 634, L129

Stefanon, M. \& Marchesini, D. 2011, ArXiv e-prints

Stott, J. P., Smail, I., Edge, A. C., Ebeling, H., Smith, G. P., Kneib, J.-P., \& Pimbblet, K. A. 2007, ApJ, 661, 95

Strazzullo, V., Rosati, P., Pannella, M., Gobat, R., Santos, J. S. Nonino, M., Demarco, R., Lidman, C., Tanaka, M., Mullis, C. R., Nuñez, C., Rettura, A., Jee, M. J., Böhringer, H., Bender, R., Bouwens, R. J., Dawson, K., Fassbender, R., Franx, M., Perlmutter, S., \& Postman, M. 2010, A\&A, 524, A17

Strazzullo, V., Rosati, P., Stanford, S. A., Lidman, C., Nonino, M., Demarco, R. Eisenhardt, P. E., Ettori, S., Mainieri, V., \& Toft, S. 2006, A\&A, 450, 909

Suzuki, N., Rubin, D., Lidman, C., Aldering, G., Amanullah, R., Barbary, K., Barrientos, L. F., Botyanszki, J., Brodwin, M., Connolly, N., Dawson, K. S., Dey, A., Doi, M., Donahue, M., Deustua, S., Eisenhardt, P., Ellingson, E., Faccioli, L., Fadeyev, V., Fakhouri, H. K., Fruchter, A. S., Gilbank, D. G., Gladders, M. D., Goldhaber, G., Gonzalez, A. H., Goobar, A., Gude, A., Hattori, T., Hoekstra, H., Hsiao, E., Huang, X., Ihara, Y., Jee, M. J., Johnston, D., Kashikawa, N., Koester, B., Konishi, K., Kowalski, M., Linder, E. V., Lubin, L., Melbourne, J., Meyers, J., Morokuma, T., Munshi, F., Mullis, C., Oda, T., Panagia, N., Perlmutter, S., Postman, M., Pritchard, T., Rhodes, J., Ripoche, P., Rosati, P., Schlegel, D. J., Spadafora, A., Stanford, S. A., Stanishev, V., Stern, D., Strovink, M., Takanashi, N., Tokita, K., Wagner, M., Wang, L., Yasuda, N., Yee, H. K. C., \& Supernova Cosmology Project, T. 2012, ApJ, 746, 85

Tran, K.-V. H., Papovich, C., Saintonge, A., Brodwin, M., Dunlop, J. S., Farrah, D., Finkelstein, K. D., Finkelstein, S. L., Lotz, J., McLure, R. J., Momcheva, I., \& Willmer, C. N. A. 2010, ApJ', 719 , L126

Villar, V., Gallego, J., Pérez-González, P. G., Barro, G., Zamorano, J., Noeske, K., \& Koo, D. C. 2011, ApJ, 740, 47

Wilman, D. J., Oemler, Jr., A., Mulchaey, J. S., McGee, S. L., Balogh, M. L., \& Bower, R. G. 2009, ApJ, 692, 298 